Check for updates

Cite this: RSC Adv., 2019, 9, 17179

Accepted 23rd May 2019

DOI: 10.1039/c9ra02612f

rsc.li/rsc-advances

\title{
Visible-light-triggered supramolecular valves based on $\beta$-cyclodextrin-modified mesoporous silica nanoparticles for controlled drug release $\uparrow$
}

\author{
Qing Bian, (D) *a Zhaolu Xue, ${ }^{\mathrm{b}}$ Po Sun, ${ }^{\mathrm{a}}$ Kejing Shen, ${ }^{\mathrm{a}}$ Shangbing Wang ${ }^{\mathrm{a}}$ \\ and Juanying Jia*a
}

A high-efficiency drug delivery system has been successfully constructed based on visible-light triggered binding and releasing between tetra-ortho-methoxy-substituted azobenzene (mAzo) and $\beta$-cyclodextrin $(\beta-C D)$ modified mesoporous silica nanoparticles. The drug releasing efficiency is calculated to be $56 \%$. The high-efficiency visible-light-triggered drug delivery system may afford great potential for cancer therapy.

Drug-delivery systems nowadays find a great number of advanced applications in reducing drug toxicity and enhancing therapeutic efficacy. ${ }^{1-3}$ Internal or external stimuli, such as enzyme, ${ }^{4}$ temperature, ${ }^{5} \mathrm{pH}^{6,7}$ redox, ${ }^{8}$ and light, ${ }^{9,10}$ have attracted great attention in controlling drug release at target sites of diseases. Among the above stimuli, light is an especially attractive external stimulus because of its possible achievement of spatial and temporal control. However, most of the UV-lighttriggered drug-delivery system have achieved limited success in vitro or in vivo mainly because of the UV light may damage to biological samples and living tissues. ${ }^{11-14}$ Compared with UV light, visible light is much less damaging to biological specimens and living tissues, which has been widely applied in developing drug delivery systems for the clinical applications. ${ }^{15-17}$

Tetra-ortho-methoxy-substituted azobenzene (mAzo) is a novel visible-responsive chromophore. ${ }^{18-21}$ Different from the conventional UV-responsive azopolymer, the mAzo-containing polymer can be isomerized the trans-isomeric state to cisisomeric state by green light irradiation, and the reverse photoswitching can be accomplished with blue light, so bidirectional photoswitching between thermally stable isomers is possible without using UV light at all. ${ }^{22,23}$ Besides the visible light responsiveness, mAzo is also sensitive to $\mathrm{pH}$ due to the protonation of the azo group to give an azonium protomer. ${ }^{24,25}$ Based on the visible responsive mAzo/ $\beta$-CD supramolecular interactions, light-responsive supramolecular hydrogels for controlled protein release from deep tissues were designed. ${ }^{26}$

\footnotetext{
${ }^{a}$ Analysis and Testing Central Facility of Anhui University of Technology, Maanshan 243032, China. E-mail: bq66bq@126.com

${ }^{b}$ Research Center of Modern Surface, Interface Engineering of Anhui University of Technology, Maanshan 243032, China

$\dagger$ Electronic supplementary information (ESI) available: Experimental details, synthetic routes, UV-visible absorption spectra. See DOI: 10.1039/c9ra02612f
}

Based on the visible-light responsive performance of mAzo, a visible light responsive random copolymer consisting of mAzo-containing acrylate units and acrylic acid units has been prepared, and the amphiphilic polymer can self-assemble into nanoparticles in water which can loaded model drugs. ${ }^{27}$ However, at light irradiation, most of the loaded model drugs in the self-assembled nanoparticles could not be released because of the small polarity changes of mAzo.

Mesoporous silica nanoparticles (MSNs) with large surface area are generally considered as ideal drug delivery candidates due to their excellent biocompatibility and mechanical and chemical stability. ${ }^{28,29}$ Mesoporous silica nanomaterial with stimuli-responsive capping agents has been demonstrated as one of the most promising and high-efficiency drug carriers. However, current release efficiency of the MSNs systems was so low. A visible light and $\mathrm{pH}$ responsive drug delivery system based on perylene-functionalized poly(dimethylaminoethyl methacrylates) coated MSNs has been developed, and the release efficiency would be reached about $45 \%$ when combined with visible light and $\mathrm{pH}^{30}$ mAzo-Si was grafted onto MSNs surface to constructed drug carry system, and red-lightresponsive supramolecular valves was developed by $\mathrm{mAzo} / \beta$ $\mathrm{CD}$ which could release drugs under red light irradiation, and about $38 \%$ of drugs was released. ${ }^{31}$

Herein, MSNs with a visible-light-responsive polymer which was used as a visible-light-triggered gate-keeper has been designed as one of the most promising and high efficiency drug releasing system (Scheme 1). MSNs were modified by $\beta$-CD via a ring-opening reaction. An mAzo-containing amphiphilic copolymer poly $\left\{6-\left[\left(2,6-\right.\right.\right.$ dimethoxyphenyl)azo- $4-\left(2^{\prime}, 6^{\prime}\right.$ dimethoxy $)$ phenoxy]propyl dimethylaminoethyl methacrylate-random$\operatorname{poly}(2-(N, N$-dimethylaminoethyl)methacrylate) $\}$

(AzoPDMAEMA) was synthesized by quaternization reaction and used as the visible-light-triggered gate-keeper. $p$-Coumalic acid 


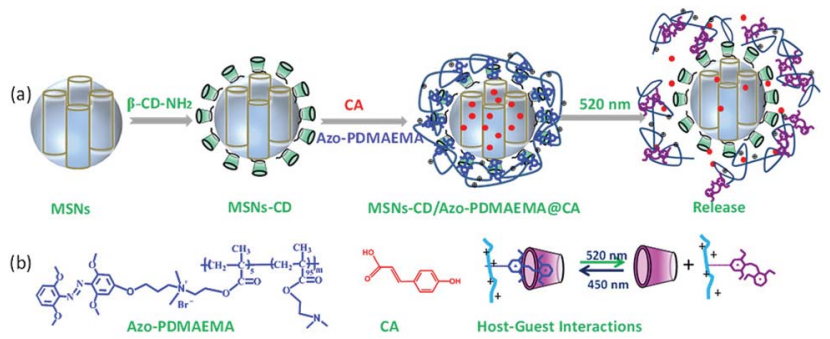

Scheme 1 (a) Schematic depiction of visible-light triggered cargo release from MSNs-CD/Azo-PDMAEMA. (b) Chemical structure of Azo-PDMAEMA and $p$-coumalic acid (CA), and schematic illustration of the supramolecular interaction between Azo-PDMAEMA and $\beta-C D$.

(CA) was used as model drug and loaded into nanopores of the MSNs. Visible-light was utilized to controlled inclusion and exclusion reaction of the Azo-PDMAEMA with $\beta$-CD on the surface of MSNs to reversibly control CA release from the MSNs or in deep tissue. Before green light irradiation, CA was loaded into the MSNs pores by free diffusion. Upon irradiation with green light, the mAzo switched from trans- to cis-isomers, and the cis-azobenzene was not recognized by $\mathrm{CD}$ due to the unmatched host-guest pairs; thus, the loaded drugs could be released. The great drug release amount of CA is calculated to be $62 \%$ which may afford great potential for cancer therapy.

The visible-light responsive copolymer Azo-PDMAEMA was synthesized according to our previous report. ${ }^{32}$ The polycation Azo-PDMAEMA was synthesized by quaternization between 2,6,2', 6'-tetramethoxy-(4-(3-hydroxypropoxy-bromide)) azobenzene (AzoOMeBr) and poly(2-( $N, N$-dimethylaminoethyl) Methacrylate) (PDMAEMA). The synthetic route, the ${ }^{1} \mathrm{H}$ NMR spectra, and the visible-light isomerization properties of the Azo-PDMAEMA were shown in Fig. S1, Fig. S2, and Fig. S3, $\dagger$ respectively. The number-average molecular weight $\left(M_{\mathrm{n}}\right)$ and PDI of the PDMAEMA were $2.08 \times 10^{4} \mathrm{~g} \mathrm{~mol}^{-1}$ and 1.19 respectively, as determined by GPC, as shown in Fig. S4. $\dagger$ The degree of functionalization (DF) of the Azo-PDMAEMA was measured by UV-vis spectroscopy, which was calculated to be $20.75 \%$, shown in Fig. S5. $\dagger$ MSNs as drug delivery systems, were prepared according to the previous literature. ${ }^{28,30}$ MSNs were functionalized with $\beta$-CD- $\mathrm{NH}_{2}$ to obtain MSNs-CD. The Fourier transform infrared (FTIR) spectra for MSNs-CD (Fig. 1a, green line) shows the absorption peaks of the amide group at $1658 \mathrm{~cm}^{-1}(\nu \mathrm{C}=\mathrm{O})$ and $3353 \mathrm{~cm}^{-1}(\delta \mathrm{N}-\mathrm{H})$, which indicated that
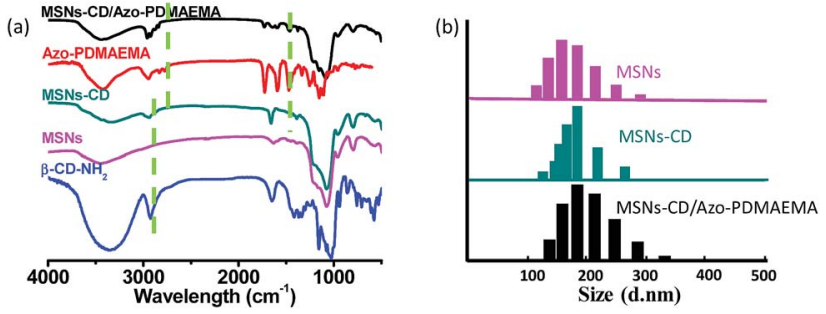

Fig. 1 (a) FTIR spectra of $\beta-C D-N_{2}$, MSNs, MSNs-CD, AzoPDMAEMA and MSNs-CD/Azo-PDMAEMA. (b) Size distributions for MSNs, MSNs-CD and MSNs-CD/Azo-PDMAEMA.
$\beta$-CD- $\mathrm{NH}_{2}$ was successfully grafted onto the MSNs. After the polymer Azo-PDMAEMA coated onto the MSNs-CD, the characteristic peaks of Azo-PDMAEMA at $1746 \mathrm{~cm}^{-1}, 2933 \mathrm{~cm}^{-1}$ which associate to $\mathrm{C}=\mathrm{O}$ and $\mathrm{C}-\mathrm{H}$ stretching vibration could be found in the spectrum of MSNs-CD/Azo-PDMAEMA, shown in Fig. 1a (black line). Moreover, the organic modification process was also further testified by dynamic light scattering (DLS) as shown in Fig. $1 \mathrm{~b}$. The hydrodynamic radius $\left(R_{\mathrm{h}}\right)$ of MSNs was approximately $175 \mathrm{~nm}$, after $\beta$-CD- $\mathrm{NH}_{2}$ modification, the $R_{\mathrm{h}}$ increases to $184 \mathrm{~nm}$, owing to the volume of the $\beta$-CD. Moreover, after Azo-PDMAEMA polycation was coated onto MSNs, the $R_{\mathrm{h}}$ increases to $187 \mathrm{~nm}$.

The model drugs $p$-coumalic acid (CA) could be effectively encapsulated into the MSNs-CD by free diffusion, and then the Azo-PDMAEMA was attached on the surface of MSNs-CD. The step-by-step functionalization processes were also confirmed by Transmission Electron Microscopy (TEM), small-angle X-ray diffraction (SA-XRD), and $\mathrm{N}_{2}$ adsorption-desorption isotherm.

TEM images showed that MSNs, MSNs-CD and MSNs-CD/ Azo-PDMAEMA@CA were two-dimensional hexagonal nanoparticles with an average diameter of $150 \pm 30 \mathrm{~nm}$ respectively. There were no obvious differences in shape and average diameter between MSNs and MSNs-CD, and the pore size of the mesoporous structures was $\sim 2.5 \mathrm{~nm}$ (Fig. 2a and b). However, the mesoporous structure of MSNs-CD/Azo-PDMAEMA@CA became obscure, indicating that $\mathrm{CA}$ occupied the nanopores and surface coverage of the monolayered supramolecular nanovalves. Moreover, the mesoporous structure was investigated by SA-XRD. In Fig. 2d, MSNs and MSNs-CD display the obvious diffraction peak at about $2.2^{\circ}$ with low ordered degree. That is consistent with the TEM images, exhibiting the twodimensional hexagonal mesoporous structure of the silica shell. Compared with the diffraction intensity of MSNs and MSNs-CD, the decreased intensity of MSNs-CD/AzoPDMAEMA@CA is ascribed to the cargo loading and the organic functional coating.

The $\mathrm{N}_{2}$ adsorption-desorption isotherms were carried out to investigate the mesoporous structure of MSNs, MSNs-CD, and MSNs-CD/Azo-PDMAEMA@CA, as shown in Fig. 2e. MSNs
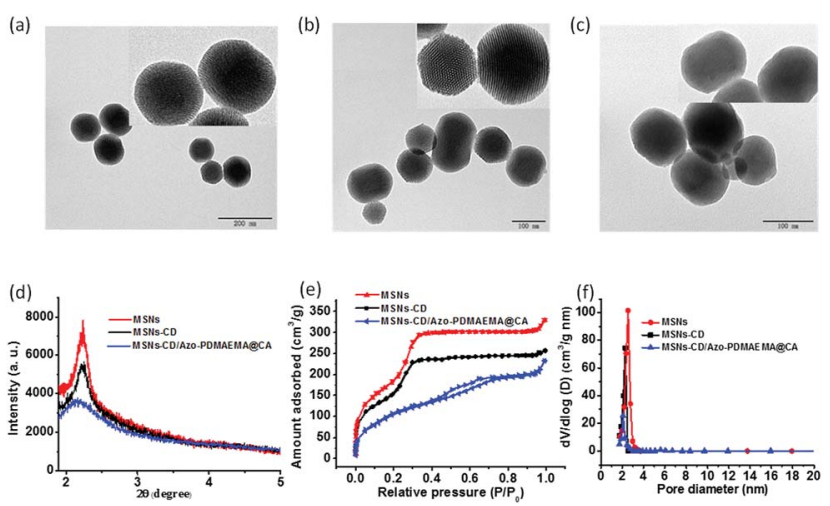

Fig. 2 TEM images of MSNs (a), MSNs-CD (b) and MSNs-CD/AzoPDMAEMA@CA (c). XRD patterns (d), nitrogen adsorption-desorption isotherms (e), and BJH pore size distribution (f) of MSNs, MSNs-CD and MSNs-CD/Azo-PDMAEMA@CA, respectively. 
showed typical $\mathrm{H}^{1}$-hysteresis loops, indicating that MSNs are mesoporous materials. The Brunauer-Emmett-Teller (BET) surface area of MSNs was $886.0518 \pm 12 \mathrm{~m}^{2} \mathrm{~g}^{-1}(n=3)$. The $\mathrm{N}_{2}$ adsorption-desorption isotherms of MSNs-CD were similar to those of MSNs, and the BET surface area of MSNs-CD (734.2583 $\left.\pm 20 \mathrm{~m}^{2} \mathrm{~g}^{-1}\right)$ was almost the same as that of MSNs. After loading with $\mathrm{CA}$, much weaker $\mathrm{N}_{2}$ absorption was observed for the MSNs-CD/Azo-PDMAEMA@CA, and the BET surfaces area changed to $299.432 \pm 15 \mathrm{~cm}^{2} \mathrm{~g}^{-1}$, indicating the successful loading of CA. The Barrett-Joyner-Halanda (BJH) pore sizes of the MSNs and MSNs-Azo were similar (2.2 to $2.5 \mathrm{~nm}$ ) to those measured using TEM (Fig. 2f). For the MSNs-CD/AzoPDMAEMA@CA, the peak of BJH pore size distribution was weak, which was coincided to the TEM and BET results.

Green light $(520 \mathrm{~nm})$ triggered the opening of $\mathrm{mAzo} / \beta-\mathrm{CD}$ valves and the cargo release. To evaluate the light-triggered properties of the cargo release from the MSNs-CD/AzoPDMAEMA@CA, UV-vis spectrophotometer was employed to monitor the absorption spectra upon irradiation of green (520 $\mathrm{nm}$ ) light and without light irradiation. Two equal samples of MSNs-CD/Azo-PDMAEMA@CA were dissolved in $1 \mathrm{~mL}$ of deionized water and introduced into a dialysis membrane (MWCO: $3500 \mathrm{Da}$ ). The released CA outside of the dialysis membrane was sampled at defined time and assayed by UV-vis absorption spectroscopy. Fig. 3a shows the UV-vis absorption spectral change of the loaded CA upon green light irradiation for different times, from which it can be seen that a remarkable increase of the absorption band at $285 \mathrm{~nm}$ was observed with increasing green irradiation time. With the extension of release time, the release amounts increase. The release amount increased to $56 \%$, and the release can reach equilibrium after 30 min. While, the absorption band of CA changed little when in dark, as shown in Fig. 3b, as indicated no leakage of CA. As shown in Fig. 3c, the great release amounts upon green light irradiation (56\%) are derived from the host-guest interaction between mAzo and $\beta$-CD to induce more CA to diffuse outside. A control experiment without green light irradiation was also carried to reveal just $7 \%$ of CA was leached into the aqueous solution even after $50 \mathrm{~h}$. This result strongly demonstrated that MSNs-CD/Azo-PDMAEMA@CA would faster release cargos under green light irradiation.

In contrast to the zeta potential of $-26.1 \mathrm{mV}$ for the MSNs$\mathrm{CD}$, the value of zeta potential for the MSNs-CD/AzoPDMAEMA@CA increased to $63.4 \mathrm{mV}$, shown in Fig. S8, $\dagger$ since the polymer Azo-PDMAEMA was positively charged. After green light irradiation, the zeta potential decreased to

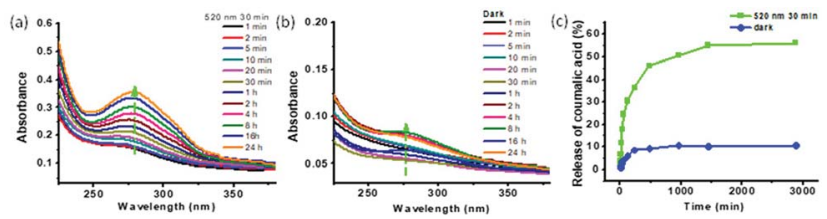

Fig. 3 UV-vis spectra of the CA in aqueous solution at different times: (a) irradiated with green light, (b) without green light irradiation. (c) Release of CA from MSNs-CD/Azo-PDMAEMA@CA with and without green light irradiation.

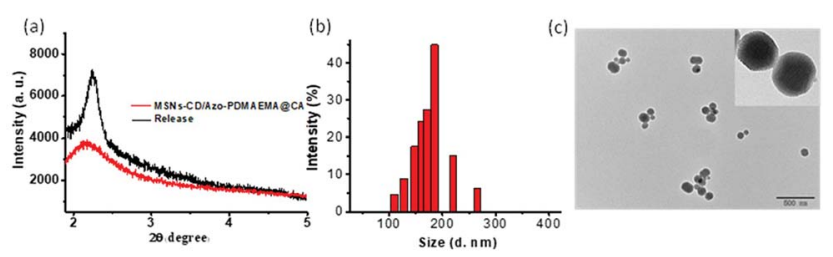

Fig. 4 (a) The small-angle XRD patterns of MSNs-CD/Azo-PDMAE$M A @ C A$ before and after green light irradiation. Size distributions (b) and TEM image (c) for MSNs-CD/Azo-PDMAEMA@CA after green light irradiation.

$-13.2 \mathrm{mV}$, indicated the drug released by deshielding of the polymer shell. Moreover, after green light irradiation, the mesoporous characteristic of MSNs-CD/Azo-PDMAEMA@CA was also confirmed by SAXRD (shown in Fig. 4a, black curve), from which it can be seen that the MSNs-CD/AzoPDMAEMA@CA still exhibited uniform mesoporous nanostructure compared with MSNs-CD/Azo-PDMAEMA@CA before green light irradiation. This result indicated the success release of CA and polymer Azo-PDMAEMA from MSNs-CD after green light irradiation. The nanostructure of the MSNs-CD/AzoPDMAEMA@CA after green light irradiation was also visualized by TEM. Fig. 4c shows that the porous structure of the nanoparticles became obvious, suggesting the surface coverage of the polymer Azo-PDMAEMA have been released after green light irradiation, and the diameter is about $185 \mathrm{~nm}$, which corresponds to the result of a $R_{\mathrm{h}}$ indicated by DLS shown in Fig. 4b. The FTIR spectra for MSNs-CD/Azo-PDMAEMA after green light irradiation was also shown in Fig. $\mathrm{S} 7, \dagger$ the characteristic peaks of Azo-PDMAEMA at $1746 \mathrm{~cm}^{-1}, 2933 \mathrm{~cm}^{-1}$ which associate to $\mathrm{C}=\mathrm{O}$ and $\mathrm{C}-\mathrm{H}$ stretching vibration disappeared which also indicated the success release of Azo-PDMAEMA from MSNs-CD after green light irradiation.

In conclusion, visible-light triggered MSNs using mAzo/ $\beta$-CD supramolecular valves for cargo release has been demonstrated. MSNs were used as drug carriers, and a model drug (CA) could be loaded via free diffusion. Visible light triggered supramolecular valves constructed by $\mathrm{mAzo} / \beta-\mathrm{CD}$ were grafted on the MSNs surface. The green light could transform the isomerism of $\beta$-CD the mAzo groups from trans to cis conformation, which result in detachment of Azo-PDMAEMA from modified MSNs, triggering the cargo release. The great drug release amount of CA is calculated to be $56 \%$ which may afford great potential for cancer therapy. We envision that this strategy described here will provide a new option to solve the problem of premature drug release in normal MSNs and have great application for combination chemotherapy.

\section{Conflicts of interest}

There are no conflicts to declare.

\section{Notes and references}

1 S. Mura, J. Nicolas and P. Couvreur, Nat. Mater., 2013, 12, 991. 
2 T. Q. Tang, L. L. Li and D. Chen, Adv. Mater., 2012, 24, 1504. 3 J. C. Imperiale, G. B. Acosta and A. Sosnik, J. Controlled Release, 2018, 285, 106.

4 D. Lizonova, J. Muzik, M. Soltys, J. Beranek, S. G. Kazarian and F. Stepanek, Eur. J. Pharm. Biopharm., 2018, 130, 327.

5 C. Ma, Y. Shi, D. A. Pena, L. Peng and G. H. Yu, Angew. Chem., 2015, 127, 7484.

6 H. U. Kim, D. G. Choi, Y. H. Roh, M. S. Shim and K. W. Bong, Small, 2016, 25, 3463.

7 Y. Hu, C. J. Yu, H. Zhang, J. S. Wang, G. Q. Jiang and C. Y. Kan, J. Nanosci. Nanotechnol., 2017, 17, 900.

8 H. Cho, J. Bae, V. K. Garripelli, J. M. Anderson, H. W. Jun and S. Jo, Chem. Commun., 2012, 48, 6043.

9 T. Lajiunen, L. Viitala, L. S. Kontturi, T. Laaksonen, H. Liang, V. L. Elina, T. Viitala, X. L. Guével, M. Yliperttula, L. Murtomäki and A. Urtti, J. Controlled Release, 2015, 203, 85.

10 P. Xiao, J. Zhang, J. Zhao and M. H. Stenzel, Prog. Polym. Sci., 2017, 74, 1.

11 C. Bao, B. Ma, J. Liu, Z. Wu, H. Zhang, Y. J. Jiang and J. Sun, Langmuir, 2016, 32, 3393.

12 J. Liu, W. Bu, L. Pan and J. Shi, Angew. Chem., Int. Ed., 2013, $\mathbf{5 2}, 4375$.

13 W. Li, J. Wang, J. Ren and X. Qu, J. Am. Chem. Soc., 2014, 136, 2248.

14 S. Chen, Y. J. Gao, Z. Q. Cao, B. Wu, L. Wang, H. Wang, Z. M Dang and G. J. Wang, Macromolecules, 2016, 49, 7490.

15 L. Stricker, E. C. Fritz, M. Peterlechner, N. L. Doltsinis and B. J. Ravoo, J. Am. Chem. Soc., 2016, 138, 4547.

16 M. Dong, A. Babalhavaeji, S. Samanta, A. A. Beharry and G. A. Woolley, Acc. Chem. Res., 2015, 48, 2662.
17 G. J. Wang, J. Dong, T. Yuan, J. Zhang, L. Wang and H. Wang, Macromol. Biosci., 2016, 16, 990.

18 A. A. Beharry, O. Sadovski and G. A. Woolley, J. Am. Chem. Soc., 2011, 133, 19684.

19 S. Samanta, T. M. Mccormick, S. K. Schmidt, D. S. Seferos and G. A. Woolley, Chem. Commun., 2013, 49, 10314.

20 G. J. Wang, D. Yuan, T. Yuan, J. Dong, N. Feng and G. Han, J. Polym. Sci., Part A: Polym. Chem., 2015, 53, 2768.

21 P. Weis, D. Wang and S. Wu, Macromolecules, 2016, 49, 6368.

22 A. Beharry, O. Sadovski and G. Woolley, J. Am. Chem. Soc., 2011, 133, 19684.

23 S. Samanta, T. Mccormick, S. Schmidt, D. Seferos and G. Woolley, Chem. Commun., 2013, 49, 10314.

24 S. Samanta, A. Beharry, O. Sadovski, T. Mccormick, A. Babalhavaeji, V. Tropepe and G. Woolley, J. Am. Chem. Soc., 2013, 26, 9777.

25 M. Hansen, M. Lerch, W. Szymanski and B. Feringa, Angew. Chem., 2016, 43, 13712.

26 D. Wang, M. Wagner, H. J. Butt and S. Wu, Soft Matter, 2015, 11, 7656.

27 G. Wang, D. Yuan, T. Yuan, N. Feng and G. Han, J. Polym. Sci., Part A: Polym. Chem., 2015, 23, 2768.

28 Q. L. Li, L. Wang, X. Qiu, Y. Sun, P. Wang, Y. Liu, F. Li, A. Qi, H. Gao and Y. Yang, Polym. Chem., 2014, 5, 3389.

29 T. Wang, G. Sun, M. Wang, B. Zhou and J. Fu, ACS Appl. Mater. Interfaces, 2015, 7, 21295.

30 G. Wang, J. Dong, T. Yuan, J. Zhang, L. Wang and H. Wang, Macromol. Biosci., 2016, 16, 990.

31 D. Wang and S. Wu, Langmuir, 2016, 32, 632.

32 Q. Bian, M. Jin, S. Chen, L. Xu, S. Wang and G. Wang, Polym. Chem., 2017, 8, 5525. 